\title{
Optical Beams in Nonlocal Nonlinear Media
}

\author{
W. KRÓLIKOWSKI
}

Australian Photonics Cooperative Research Centre, Laser Physics Centre Research School of Physical Science and Engineering

The Australian National University, Canberra ACT 0200, Australia

$$
\text { O. BANG }
$$

Department of Mathematical Modelling

Technical University Denmark, Building 305/321, 2800 Kgs. Lyngby, Denmark

\section{J. WYLLER}

Department of Mathematical Sciences, Agricultural University of Norway P.O. Box 5065, 1432 Ås, Norway

\section{AND J.J. RASMUSSEN}

Risø National Laboratory, Optics and Fluid Dynamics Department, OFD - 128 P.O. Box 49, 4000 Roskilde, Denmark

We discuss propagation of optical beams in nonlocal Kerr-like media with the nonlocality of general form. We study the effect of nonlocality on modulational instability of the plane wave fronts, collapse of finite beams and formation of spatial solitons.

PACS numbers: 42.65.Tg, 42.65.Jx

\section{Introduction}

Let us consider a phenomenological model of nonlocal nonlinear Kerr type media, in which the refractive index change $\Delta n$ induced by a beam with intensity $I(x, z)$ can be represented in general form as

$$
\Delta n(I)= \pm \int_{-\infty}^{\infty} R\left(x^{\prime}-x\right) I\left(x^{\prime}, z\right) \mathrm{d} x^{\prime},
$$

where the positive (negative) sign corresponds to a focusing (defocusing) nonlin- 
earity and $x$ and $z$ denote transverse and propagation coordinates, respectively. The real, localized, and symmetric function $R(x)$ is the response function of the nonlocal medium, whose width determines the degree of nonlocality. For a singular response, $R(x)=\delta(x)$, the refractive index change becomes a local function of the light intensity, $\Delta n(I)= \pm I(x, z)$, i.e. the refractive index change at a given point is solely determined by the light intensity at that very point. With increasing width of $R(x)$ the light intensity in the vicinity of the point $x$ also contributes to the index change at that point (see Fig. 1 where various degrees of nonlocality determined by the width of the nonlocal response function are shown). In the limit of a highly nonlocal response Snyder and Mitchell showed that the beam evolution was described by the simple equation for a linear harmonic oscillator [1]. The influence of nonlocality of the nonlinear response on the dynamics of beams was illustrated for the special logarithmic nonlinearity, which allows exact analytical treatment [2].

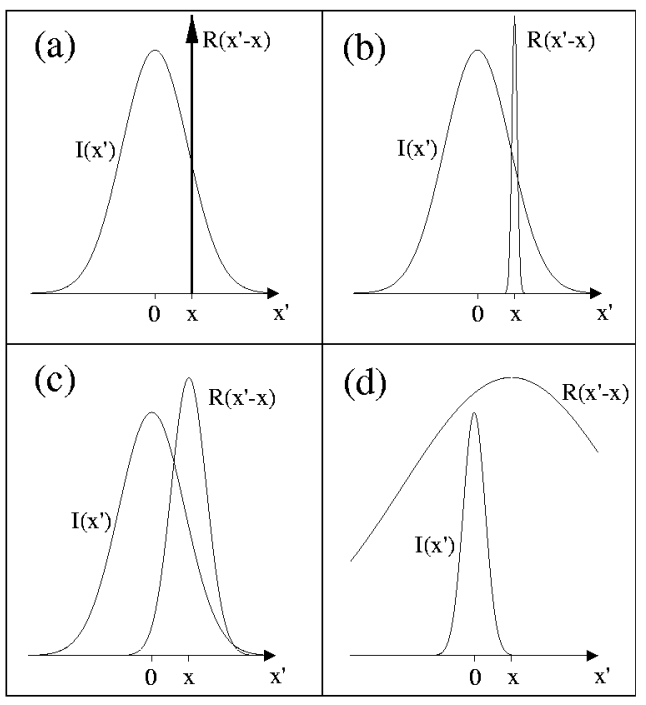

Fig. 1. Different degrees of nonlocality, as given by the width of the response function $R(x)$ and the intensity profile $I(x)$. Shown is the local (a), the weakly nonlocal (b), the general (c), and the strongly nonlocal (d) response.

While Eq. (1) is a phenomenological model, it nevertheless describes several real physical situations. Possible physical mechanisms responsible for this type of nonlinear response includes various transport effects, such as heat conduction in materials with thermal nonlinearity [3-5], diffusion of molecules or atoms accompanying nonlinear light propagation in atomic vapours [6], and drift and/or diffusion of photoexcited charges in photorefractive materials [7, 8]. A highly nonlocal nonlinearity of the form (1) has also been identified in plasmas [9-13], liquid crystals 
[14] and it appears as a result of many-body interaction processes in the description of Bose-Einstein condensates [15]. It has been also shown recently that $\chi^{(2)}$ solitons generated in the process of second harmonic generation are equivalent to nonlocal solitons with exponential response function [16].

Even though it is quite apparent in some physical situations that the nonlinear response in general is nonlocal (as in the case of thermal lensing), the nonlocal contribution to the refractive index change was often neglected [17, 18]. This is justified if the spatial scale of the beam is large compared to the characteristic response length of the medium (given by the width of the response function). However, for very narrow beams the nonlocality can be of crucial importance and has to be taken into account. For instance, it has been shown theoretically that a weak nonlocal contribution arrests collapse (catastrophic self-focusing) of high power optical beams in a self-focusing medium and leads to the formation of stable 2D (diffracting in two transverse dimensions) solitons $[12,13,19,20]$.

Some of the consequences of nonlocality in the nonlinear response have been observed experimentally. Suter and Blasberg reported stabilization of 2D solitary beams in atomic vapours due to atomic diffusion, which carries excitation away from the interaction region [6]. Also, the discrepancy between the theoretical model of dark solitons and that observed experimentally in a medium with thermal nonlinearity has been associated with nonlocality of the nonlinearity $[17,18]$.

Here, we summarize our recent works on propagation of optical beams in nonlinear nonlocal media of the general form (1). In Sec. 2 we will discuss the effect of nonlocality on modulational instability of plane wave. Section 3 will be devoted to the role of nonlocality in arresting the collapse of finite beams. In Sec. 4 we discuss propagation of 1-dimensional solitons in weakly nonlocal media. We summarize our findings in Sec. 4.

\section{Modulational instability}

Modulational instability (MI) constitutes one of the most fundamental effects associated with wave propagation in nonlinear media. It signifies the exponential growth of a weak perturbation of the amplitude of the wave as it propagates. The gain leads to amplification of side bands, which breaks up the otherwise uniform wave front and generates fine localized structures (filamentation). Thus it may act as a precursor for the formation of bright spatial solitons. Conversely the generation of dark spatial solitons requires the absence of MI of the constant intensity background.

The phenomena of MI have been identified and studied in various physical systems, such as fluids [21], plasma [22], nonlinear optics [23, 24], discrete nonlinear systems, such as molecular chains [25] and Fermi-resonant interfaces and waveguide arrays [26], etc. It has been shown that MI is strongly affected by various mechanisms present in nonlinear systems, such as higher order dispersive terms in 
the case of optical pulses [27], saturation of the nonlinearity [28], and coherence properties of optical beams [29].

We will consider an optical beam propagating along the $z$-axis, with the scalar amplitude of the electric field expressed as

$$
E(x, z)=\psi(x, z) \exp (\mathrm{i} K z-\mathrm{i} \Omega t)+\text { c.c. },
$$

where $K$ is the wave number, $\Omega$ is the optical frequency and $\psi(x, z)$ is the slowly varying amplitude. Substituting Eqs. (1) and (2) into Maxwell's equations we obtain the nonlocal nonlinear Schrödinger (NLS) equation

$$
\mathrm{i} \partial_{z} \psi+\frac{1}{2} \partial_{x}^{2} \psi+s \psi \int_{-\infty}^{\infty} R\left(x^{\prime}-x\right) I\left(x^{\prime}, z\right) \mathrm{d} x^{\prime}=0
$$

where $I(x, z)=|\psi(x, z)|^{2}$ is the intensity of the beam. The model (3) permits plane wave solutions of the form

$$
\psi(x, z)=\sqrt{\rho_{0}} \exp \left(\mathrm{i} k_{0} x-\mathrm{i} \omega_{0} z\right), \quad \rho_{0}>0,
$$

where $\rho_{0}, k_{0}$, and $\omega_{0}$ are linked through the nonlinear dispersion relation

$$
\omega_{0}=\frac{1}{2} k_{0}^{2}-s \rho_{0},
$$

which is the same as for the standard local NLS equation

$$
\mathrm{i} \partial_{z} \psi+\frac{1}{2} \partial_{x}^{2} \psi+s|\psi|^{2} \psi=0
$$

Next, let us carry out a linear stability analysis of the plane wave solutions (4). Let us assume that

$$
\psi(x, z)=\left[\sqrt{\rho_{0}}+a_{1}(x, z)\right] \exp \left(\mathrm{i} k_{0} x-\mathrm{i} \omega_{0} z\right),
$$

where $a_{1}(x, z)$ is a small complex perturbation. Inserting this expression into the nonlocal NLS equation (3) and linearizing around the solution (4) yields the evolution equation for the perturbation

$$
\mathrm{i} \partial_{z} a_{1}+\frac{1}{2} \partial_{x}^{2} a_{1}+2 s \rho_{0} \int_{-\infty}^{\infty} R\left(x^{\prime}-x\right) \operatorname{Re}\left[a_{1}\left(x^{\prime}, x\right)\right] \mathrm{d} x^{\prime}=0 .
$$

After separating the real and imaginary part of the perturbation and applying the Fourier transform to the resulting system of differential we look for its solution in the form $\propto \exp (\lambda z)$, where $\lambda$ is the so-called growth rate. The sign of $\lambda$ determines stability of the solution. For positive $\lambda$ the perturbation grows during propagation indicating instability. The growth rate is found as an eigenvalue of the resulting system of equations for the spectrum of the perturbation. It is given by the following relation [30]:

$$
\lambda^{2}=-k^{2} \rho_{0}\left[\alpha k^{2}-s \widehat{R}(k)\right],
$$

where $k$ denotes spatial frequency, $\widehat{R}(k)$ is the Fourier spectrum of $R(x)$ and we have defined the parameter $\alpha$ as 


$$
\alpha=1 /\left(4 \rho_{0}\right) .
$$

Let us notice that if the response function $R(x)$ is real and symmetric then so is the Fourier spectrum of $R(x)$, i.e. $\widehat{R}(k)=\widehat{R}^{*}(k)=\widehat{R}(-k)$.

The general eigenvalue equation $(9)$ constitutes the main result of our analysis. First of all we notice that $\widehat{R}(0)=1$, since we assumed that the response function is normalized to unity $\int_{-\infty}^{\infty} R(x) \mathrm{d} x=1$.

The well-known modulational instability (stability) result for the standard local NLS equation (6) is easily recovered from the general eigenvalue equation (9) by setting $R(x)=\delta(x)$, where $\delta(x)$ is the Dirac delta function. We get

$$
\lambda^{2}=-k^{2} \rho_{0}\left(\alpha k^{2}-s\right),
$$

where $s=+1(s=-1)$ yields instability (stability).

For a focusing nonlinearity $(s=+1)$ we therefore always have, by continuity, that $\alpha k^{2}-s \widehat{R}(k)<0$, and thus $\lambda^{2}>0$, in a certain wave-number band symmetrically centred about the origin, where $k$ is sufficiently small. It follows that we always will have (long wave) MI in the focusing case, independently of the details in the behaviour of the response function. The nonlocality tends to suppress the instability, but can never eliminate it completely. In Fig. 2 we illustrate the growth rate of the MI in case of nonlocal response in the form of Gaussian function $\left(R(x)=\exp \left(-x^{2} / \sigma^{2}\right) / \sqrt{\pi \sigma}\right)$. The suppressing effect of the nonlocality (which increases with $\sigma$ ) is clearly visible.

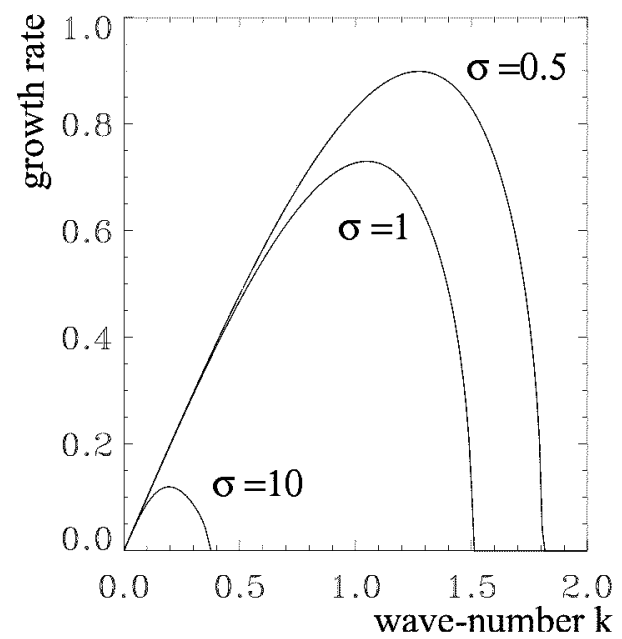

Fig. 2. MI gain profiles for $\rho_{0}=1$ in self-focusing $(s=1)$ nonlocal Kerr media with a Gaussian response profile (after [30]).

In contrast, in the defocusing case $(s=-1)$, the stability properties depend in a sensitive way on the response function. For any strictly positive continuous response profile all plane waves are stable for any degree of nonlocality. However, 


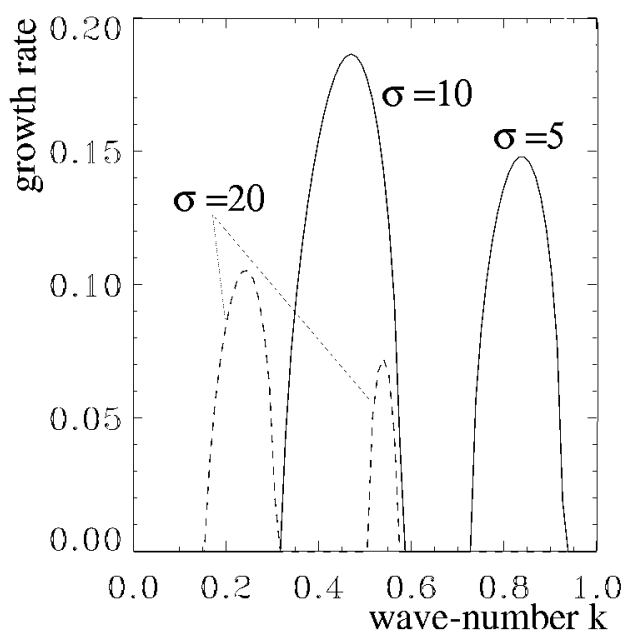

Fig. 3. MI gain profiles for $\rho_{0}=1$ in self-defocusing $(s=-1)$ nonlocal media with a rectangular response function (after [30]).

exotic profiles with jump-discontinuities like the rectangular response, may actually promote MI when the intensity is sufficiently high. Figure 3 shows the growth rate of the $\mathrm{MI}$ in self-defocusing case for the rectangular response function

$$
R(x)=\left\{\begin{array}{ccc}
\frac{1}{2 \sigma} & \text { for } & |x| \leq \sigma \\
0 & \text { for } & |x|>\sigma
\end{array}\right.
$$

Development of the high frequency instability associated with negative sign of the spectrum of the nonlocal response function, is evident.

\section{Beam collapse in nonlocal medium}

Wave collapse is a phenomenon well known in the theory of wave propagation (or evolution) in nonlinear focusing media. It refers to the situation when strong self-focusing of wave leads to catastrophic increase (blow-up) of its intensity over finite time (or space) interval [31-33]. Wave collapse has been observed in plasma waves [34], electromagnetic waves or laser beams [35], Bose-Einstein condensates (BEC's) or matter waves [36], and even capillary-gravity waves on deep water [37]. Besides wave theory the effect of collapse has been also well known in the field of astrophysics. Here the gravitational attraction playing the same role as self-focusing of electromagnetic wave, tend to compress star leading to its collapse and formation of another object such as, for instance, black hole [38, 39].

Most commonly the collapse has been discussed in the context of the, so-called, nonlinear Schrödinger equation (NLS) [40] which has been widely used to model great variety of phenomena ranging from classical optics, e.g. paraxial propagation of optical beams in nonlinear media to matter waves such as temporal evolution 
of the wave packets in the Bose-Einstein condensates. Strictly speaking, the existence of the collapse is an artefact of the model equation and signals the limit of its applicability. It is expected that in the vicinity of the collapse regime some additional physical processes will come to play and stop the blow-up [31-33]. Nevertheless, "collapse-like" (or quasicollapse) dynamics can still occur in the real physical systems when nonlinearity leads to strong energy localization. In fact, recent experiments with ultracold gases provided clear signatures of the collapse-like dynamics of the atomic condensates [36, 41, 42].

There have been few attempts to determine the role of nonlocality in development of the collapse. Turitsyn was the first to show the arrest of the collapse for particular choice of the nonlocal nonlinear response [19]. The analysis of the collapse conditions in case of general model of nonlocality is difficult and so far has been carried out only numerically [43]. Here we present analytical approach to beam collapse in nonlocal media [44].

Let us consider evolution of wave field $\psi=\psi(\boldsymbol{r})=\psi(\boldsymbol{r}, \tau)$ described by the nonlocal nonlinear Schrödinger equation of general form

$$
\mathrm{i} \frac{\partial \psi}{\partial \tau}+\nabla^{2} \psi-V(r) \psi+N(I) \psi=0,
$$

where $V=V(\boldsymbol{r})$ is an external (linear) confining potential, $I=I(\boldsymbol{r})=$ $I(\boldsymbol{r}, \tau)=|\psi|^{2}, \tau$ is the evolution coordinate and $\boldsymbol{r}=\left(r_{1}, r_{2}, r_{3}\right)$ spans a $D$-dimensional "transverse" coordinate space. The nonlinear term $N=N(I)$ is represented in the general nonlocal form

$$
N(I)=\int R\left(\boldsymbol{r}^{\prime}-\boldsymbol{r}\right) I\left(\boldsymbol{r}^{\prime}\right) \mathrm{d} \boldsymbol{r}^{\prime},
$$

where we assume the response function $R(r)$ to be real, localized, nonsingular and symmetric.

For localized or periodic solutions Eqs. (13) and (14) conserve the power (in optics) or number of atoms (for BEC) $P$ and the Hamiltonian $H$,

$$
P=\int I \mathrm{~d} \boldsymbol{r}, \quad H=\int\left(|\nabla \psi|^{2}+V I-\frac{N I}{2}\right) \mathrm{d} r .
$$

In the limit when the response function is a delta-function, $R(\boldsymbol{r})=\delta(|\boldsymbol{r}|)$, the nonlinear response is local (see Fig. 1a) and simply given by

$$
N(I)=I,
$$

as in local optical Kerr media described by the conventional NLS equation and in BEC's described by the standard Gross-Pitaevskii $(\mathrm{G}-\mathrm{P})$ equation. It is in this local limit that multidimensional optical beam with a power higher than a certain critical value would experience unbounded self-focusing and collapse after a finite propagation distance [31-33]. It is also well known that BEC would collapse when the total number of atoms was larger than a critical number [15]. 
With increasing width of the response function $R(r)$ the wave intensity in the vicinity of the point $r$ also contributes to the nonlinear response at that point. In case of weak nonlocality, when $R(\boldsymbol{r})$ is much narrow than the width of the beam, one can expand $I\left(r^{\prime}\right)$ around $r^{\prime}=r$ and obtain the simplified model

$$
N(I)=I+\gamma \nabla^{2} I, \quad \gamma=\frac{1}{2} \int r^{2} R(r) \mathrm{d} \boldsymbol{r},
$$

where a positive definite $\gamma$ measures the width of the nonlocal response.

In weakly nonlocal media with the nonlinearity Eq. (17) it is straightforward to show that collapse cannot occur. This was first done rigorously by Davydova and Fishchuk in the context of plasmas [13] i.e. without the confining potential. This result has been confirmed recently by Parola et al. [45], who used variational and exact numerical analysis to study stability of the BEC in a harmonic trap.

In the limit of a strongly nonlocal response much broader than the characteristic width of the wave function (see Fig. 1d), one can instead expand the response function and obtain (in the lowest order)

$$
N(I) \approx R(x) P .
$$

The evolution of optical beams in such a strongly nonlocal medium was considered in detail in [1]. Since this relation is linear, the highly nonlinear effect of collapse cannot occur.

Therefore in the two extreme limits of a weakly and highly nonlocal nonlinear response the collapse is prevented.

The stabilizing effect of nonlocality of a general degree in Kerr media was already noticed by Turitsyn [19]. He showed, for a few specific physical examples, such as Coulomb interaction $[R(\boldsymbol{r})=1 /|\boldsymbol{r}|]$ and the so-called funneling $[R(\boldsymbol{r})=$ $1 /|r|-\beta|r|]$ in $3 \mathrm{D}$, that the Hamiltonian was bounded from below for fixed power, and thus that the stationary ground-state solitary wave solutions (solitons) are stable in a Lyapunov sense. In turn this proves that these specific types of nonlocal response can suppress the collapse instability of multidimensional beams in Kerr media.

Here we consider the general case of arbitrarily shaped, nonsingular response functions and prove rigorously that the Hamiltonian is bounded from below so that collapse cannot occur.

Introducing the $D$-dimensional Fourier transform (denoted with a tilde) and its inverse it is straightforward to show that for $N(I)$ given by Eq. (14) the following relations hold [44]:

$$
|\tilde{I}(\boldsymbol{k})|=\left|\int I(\boldsymbol{r}) \mathrm{e}^{\mathrm{i} \boldsymbol{k} \cdot \boldsymbol{r}} \mathrm{d} \boldsymbol{r}\right| \leq \int I \mathrm{~d} \boldsymbol{r}=P
$$

and

$$
\int N I \mathrm{~d} \boldsymbol{r}=\frac{1}{(2 \pi)^{D}} \int \tilde{R}(\boldsymbol{k})|\tilde{I}(\boldsymbol{k})|^{2} \mathrm{~d} \boldsymbol{k} .
$$


For response functions with a positive definite spectrum, $\tilde{R}(\boldsymbol{k}) \geq 0$, we then have

$$
\int N I \mathrm{~d} \boldsymbol{r} \leq P^{2} \int \tilde{R}(\boldsymbol{k}) \mathrm{d} \boldsymbol{k}=P^{2} R_{0}
$$

where the positive parameter $R_{0}=R(0)$ is simply the response function evaluated at the centre. With the help of the relation Eq. (21) we can therefore bound the Hamiltonian as follows:

$$
H \geq\|\nabla \psi\|_{2}^{2}-\frac{1}{2} R_{0} P^{2}
$$

where $\|\psi\|_{p}^{p} \equiv \int|\psi|^{p} \mathrm{~d} r$ and we have used the fact that the minimum of $V(r)$ is zero.

The simple inequality (22) shows that for all symmetric response functions with a positive definite Fourier spectrum and a finite value at the centre, the Hamiltonian is bounded from below by the conserved quantity $\frac{1}{2} R_{0} P^{2}$, or conversely, that the gradient norm $\|\nabla \psi\|_{2}^{2}$ is bounded from above by the conserved quantity $H+\frac{1}{2} R_{0} P^{2}$. This represents a rigorous proof that a collapse with the wave-amplitude locally going to infinity cannot occur in BEC's or optical Kerr media with a nonlocal nonlinear response, for any physically reasonable response function (i.e. Gaussian, Lorentzian, sech-shaped, etc.).

The stabilising effect of the nonlocality can be further illustrated by the properties of the stationary solutions of Eqs. (13). As the simplest example we consider nonlocal optical bulk Kerr media with a Gaussian response. To capture the main (qualitative) physical effects we use the approximate, but simple variational technique [46] assuming Gaussian profile for the $\psi(r)$ function, $\psi(r)=\alpha \exp \left[-(r / \beta)^{2}\right] \exp (\mathrm{i} \lambda)$.
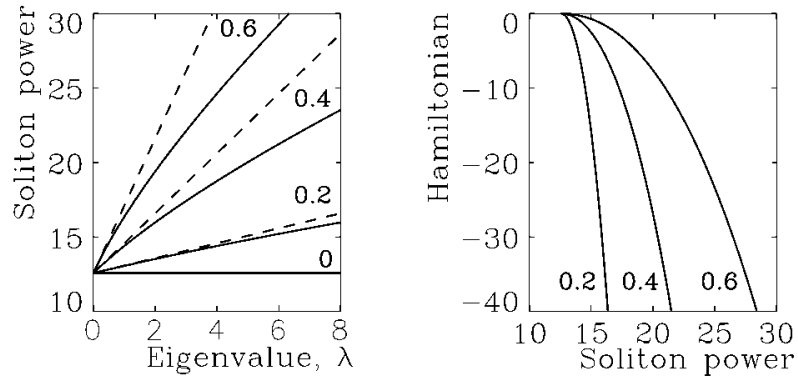

Fig. 4. 2D variational results with Gaussian response and trial function. Left: soliton power (solid) versus eigenvalue $\lambda$ for different degrees of nonlocality, $\sigma=0,0.2,0.4$, and 0.6. Dashed lines show the weakly nonlocal approximation. Right: corresponding Hamiltonian versus power diagrams (after [44]).

In Fig. 4 we show the power and Hamiltonian of the stationary solutions in $2 \mathrm{D}$. The dashed lines give the results of the weakly nonlocal approximation with $N$ given by (17), from which $\alpha^{2}=4 \lambda$ and $\beta^{2}=2 / \lambda+2 \sigma^{2}$ is found, resulting in the power 


$$
P_{s}=4 \pi\left(1+\sigma^{2} \lambda\right),
$$

where $4 \pi$ is the ( $\lambda$-independent) power of the Gaussian approximation to the soliton solutions of the standard 2D NLS equation (also the collapse threshold power), which is recovered in the local limit $\sigma=0$.

In the 2D NLS equation the collapse is a critical collapse and the stationary solutions are "only" marginally unstable with $\mathrm{d} P_{s} / \mathrm{d} \lambda=0$ [32]. Typically any perturbation will act against the self-focusing, with several effects, such as non-paraxiality and saturability, completely eliminating the possibility of a collapse [33]. This is also the case with nonlocality, as evidenced from Fig. 4 and the simplified expression (23), which shows that any finite width of the response function (non-zero value of $\sigma$ ) implies that $\mathrm{d} P_{s} / \mathrm{d} \lambda$ becomes positive definite. According to the (necessary) Vakhitov-Kolokolov (VK) criterion [47] the soliton solutions therefore (possibly) become linearly stable.

\section{Spatial solitons in nonlocal media}

In this section we demonstrate the exact analytical solutions for bright and dark spatial solitons in nonlocal media [48]. We will concentrate on propagation of 1-dimensional beams governed by the nonlocal equation (3). As shown earlier, for weak nonlocality i.e. when the response function $R(x)$ is narrow compared to the extent of the beam (see Fig. 1), this equation can be cast into the following form:

$$
\mathrm{i} \partial_{z} \psi+\frac{1}{2} \partial_{x}^{2} \psi \pm\left(|\psi|^{2}+\gamma \partial_{x}^{2}|\psi|^{2}\right) \psi=0,
$$

where $\gamma$ is defined in Eq. (17). The weak nonlocality appears thus as a perturbation to the local nonlinear refractive index change. For a single peak beam in a self-focusing medium this perturbation is of negative sign in the central part of the beam, where it serves to decrease the refractive index change. Hence, even for very narrow and sharp intensity distributions, the resulting self-induced waveguide will be wide and a smooth function of the transverse coordinates. In some sense, this is similar to saturation of the nonlinearity. One may therefore expect that certain features of solitons of Eq. (24) will be reminiscent of those exhibited by solitons in saturable nonlinear media [49]. It transpires, however, that nonlocality also results in new effects, especially for self-defocusing nonlinearities.

For a self-focusing nonlinearity the sign of the refractive index change is positive. We search for a stationary bright soliton solution to Eq. (24) of the form

$$
\psi(x, z)=u(x) \exp (\mathrm{i} \Gamma z),
$$

where the profile $u=u(x)$ is real, symmetric, and exponentially localized and the propagation constant $\Gamma>0$ is positive. For this solution Eq. (24) reduces to [48]

$$
\left(\partial_{x} u\right)^{2}=u^{2}\left(u_{0}^{2}-u^{2}\right) /\left(1+4 \gamma u^{2}\right) .
$$

This equation can be integrated leading to 


$$
x= \pm \frac{1}{u_{0}} \tanh ^{-1}\left(\frac{\kappa}{u_{0}}\right)+\sqrt{4 \gamma} \tan ^{-1}(\sqrt{4 \gamma} \kappa),
$$

where we have defined the intensity $\rho=u^{2}$ and the normalized intensity $\kappa^{2}=$ $\left(\rho_{0}-\rho\right) /(1+4 \gamma \rho)$. This implicit relation gives the profile of bright spatial solitons propagating in weakly nonlocal Kerr-like media. In the local limit, $\gamma=0$, we recover from Eq. (27) the well-known profile $u(x)=u_{0} \operatorname{sech}\left(u_{0} x\right)$ of the $1 \mathrm{D}$ bright soliton in a Kerr medium.

In Fig. 5 we show the intensity profile of the solution (27) for different values of the nonlocality parameter $\gamma$. Evidently, an increase in the degree of nonlocality results in an increase in the soliton width - nonlocality smooths out the refractive index profile thereby leading to a broadening of the beam.

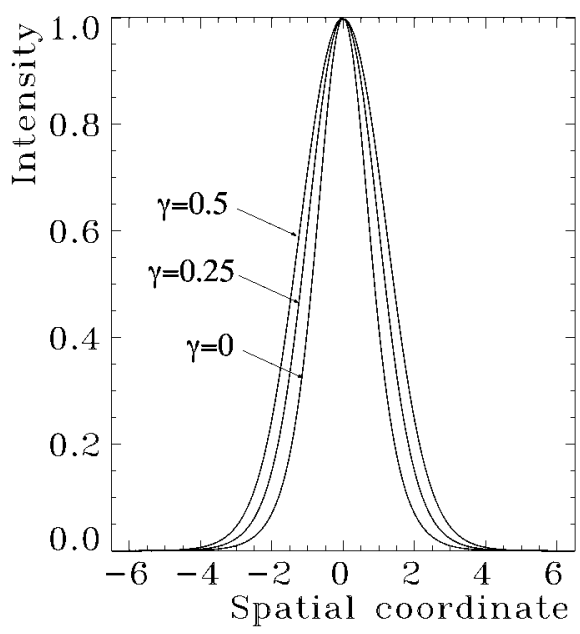

Fig. 5. Intensity profiles of bright solitons with unit amplitudes $\rho_{0}=1$ for different degrees of nonlocality $\gamma$ (after [48]).

We now consider the impact of weak nonlocality in the case of a self-defocusing nonlinearity, which corresponds to a negative sign in Eq. (23). We introduce a new spatial variable $\zeta=x-V z$, with $V$ being the soliton transverse velocity, and look for stationary solutions of the form

$$
\psi(x, z)=\sqrt{\rho(\zeta)} \exp [\mathrm{i} \Gamma z+\mathrm{i} \phi(\zeta)],
$$

where $\rho(\zeta)$ is the soliton intensity and $\phi(\zeta)$ its phase. It can be shown that the NLS equation can be integrated leading to the following solution for the soliton intensity:

$$
\pm \zeta=\frac{1}{\delta_{0}} \tanh ^{-1}\left(\frac{\delta}{\delta_{0}}\right)+\sqrt{4 \gamma} \tan ^{-1}(\sqrt{4 \gamma} \delta)
$$

which is an implicit relation between the soliton intensity $\rho$ and the spatial coordinate $\zeta$, with the normalized intensity now given by $\delta^{2}(\rho)=\left(\rho-\rho_{1}\right) /(1-4 \gamma \rho)$ 
and $\delta_{0}=\delta\left(\rho_{0}\right)$. In the above equation $\rho_{0}$ and $\rho_{1}$ denote background and minimum intensities of the dark soliton, respectively.

In Fig. 6 we present examples of the intensity profile of the dark solitons for different values of the nonlocality parameter $\gamma$. These graphs show that increasing nonlocality decreases the width of the soliton.

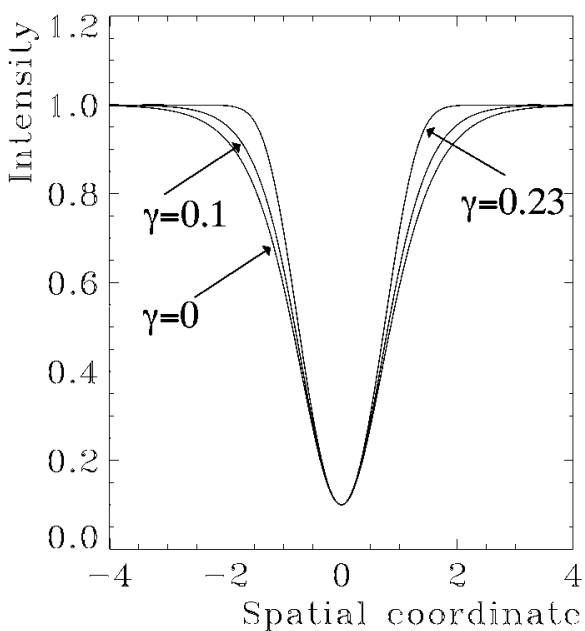

Fig. 6. Intensity profiles of dark solitons with $\rho_{0}=1$ and $\rho_{1}=0.1$ for different values of the nonlocality parameter $\gamma$ (after [48]).

An important aspect of any family of soliton solutions are their stability properties. For single peak bright solitons stability is determined by the dependence of the power

$$
P=\int_{-\infty}^{\infty}|\psi(x, z)|^{2} \mathrm{~d} x
$$

on the propagation constant $\Gamma$. Bright solitons are stable if $\mathrm{d} P / \mathrm{d} \Gamma>0$ and unstable otherwise $[50,47]$. In case of weak nonlocality power is monotonically increasing function of the propagation constant and hence these solitons are stable. In case of dark soliton the stability criterion is determined by the derivative of the so-called renormalized momentum defined as

$$
Q=\frac{\mathrm{i}}{2} \int_{-\infty}^{\infty}\left(\psi \partial_{x} \psi^{*}-\psi^{*} \partial_{x} \psi\right)\left(1-\frac{\rho_{0}}{|\psi|^{2}}\right) \mathrm{d} x .
$$

A soliton solution propagating with velocity $V$ corresponds to an extremum of the Hamiltonian for fixed momentum, $\delta(H-V Q)=0$. It has been shown that the stability criterion for dark solitons is determined by the dependence of the momentum on the velocity, $Q=Q(V)$ [51]. For dark solitons to be stable the derivative of the momentum with respect to the velocity must be positive, 


$$
\mathrm{d} Q / \mathrm{d} V>0 .
$$

Our exact solution shows that for weakly nonlocal nonlinear medium the momentum is monotonic function of soliton velocity and hence these dark solitons are stable.

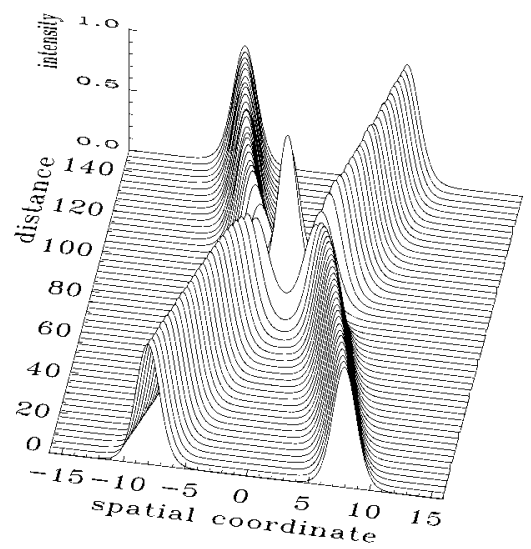

Fig. 7. Propagation and collision of bright solitons in a weakly nonlocal Kerr medium; $\gamma=0.2$ (after [48]).

In Fig. 7 we illustrate propagation and collision of two bright solitons in weakly nonlocal Kerr medium. It is evident that not only the solitons propagate in a stable manner but they also survive the collision which has clearly inelastic character.

\section{Conclusions}

In conclusion, we reviewed properties of optical beams propagating in nonlocal nonlinear Kerr-like medium. We showed that in case of self-focusing medium nonlocality always suppresses modulational instability of plane wave although it never eliminates it completely. For self-defocusing nonlinearity nonlocality can actually initiate modulational instability if the spectrum of the nonlocal response function changes sign in some range of parameters. We also showed that nonlocality stabilizes spatial solitons and arrests collapse of the multidimensional beam. Finally we presented exact analytical soliton solutions for 1-dimensional beams propagating in weakly nonlocal medium. These solitons appear to be stable.

\section{Acknowledgments}

O. Bang acknowledges support from the Danish Technical Research Council under Talent Grant No. 9800400. 


\section{References}

[1] A. Snyder, J. Mitchell, Science 276, 1538 (1997).

[2] A. Snyder, J. Mitchell, J. Opt. Soc. Am. 16, 236 (1999).

[3] J.P. Gordon, R.C. Leite, R.S. Moore, S.P. Porto, J.R. Whinnery, J. Appl. Phys. 36, 3 (1965).

[4] S. Akhmanov, D.P. Krindach, A.V. Migulin, A.P. Sukhorukov, R.V. Khokhlov, IEEE J. Quant. Electron. QE-4, 568 (1968).

[5] M. Horovitz, R. Daisy, O. Werner, B. Fischer, Opt. Lett. 17, 475 (1992); M.D. Iturbe-Castillo, J.J. Sanchez-Mondragon, S. Stepanov, Opt. Lett. 21, 1622 (1996); D. Kip, E. Krätzig, V. Shandarov, P. Moretti, Opt. Lett. 23, 343 (1998).

[6] D. Suter, T. Blasberg, Phys. Rev. A 48, 4583 (1993).

[7] S. Gatz, J. Herrmann, Opt. Lett. 23, 1176 (1998).

[8] B. Crosignani, A. Degasperis, E. DelRe, P. Di Porto, A.J. Agranat, Phys. Rev. Lett. 82, 1664 (1999).

[9] M.V. Porkolab, M.V. Goldman, Phys. Fluids 19, 872 (1976).

[10] A.G. Litvak, V.A. Mironov, G.M. Fraiman, A.D. Yunakovskii, Sov. J. Plasma Phys. 1, 31 (1975).

[11] A.G. Litvak, A.M. Sergeev, JETP Lett. 27, 517 (1978).

[12] H.L. Pecseli, J.J. Rasmussen, Plasma Phys. 22, 421 (1980).

[13] T.A. Davydova, A.I. Fishchuk, Ukr. J. Phys. 40, 487 (1995).

[14] C. Conti, M. Peccianti, G. Assanto, in: Nonlinear Guided Waves and Their Applications, OSA Technical Digest, Optical Society of America, Washington DC 2002, p. NLMD32:1-3; D.W. McLaughlin, D.J. Muraki, M.J. Shelley, X. Wang, Physica D 88, 55 (1995); M. Karpierz, Phys. Rev. E 66, 036603 (2002).

[15] F. Dalfovo, S. Giorgini, L.P. Pitaevskii, S. Stringari, Rev. Mod. Phys. 71, 463 (1999).

[16] I.V. Shadrivov, A.Z. Zharov, J. Opt. Soc. Am. B 19, 596 (2002); O. Bang, W. Krolikowski, N. Nikolov, D. Neshev, Quadratic Solitons as Nonlocal Solitons, unpublished.

[17] G.A. Swartzlander, Jr., C.T. Law, Phys. Rev. Lett. 69, 2503 (1992).

[18] B. Luther-Davies, X. Yang, Opt. Lett. 17, 1755 (1992); A. Dreischuh, G.G. Paulus, F. Zacher, F. Grasbon, H. Walther, Phys. Rev. E 60, 6111 (1999).

[19] S.K. Turitsyn, Teor. Mat. Fiz., 64, 226 (1985).

[20] S. Abe, A. Ogura, Phys. Rev. E 57, 6066 (1998).

[21] T.B. Benjamin, J.E. Feir, J. Fluid. Mech. 27, 417 (1967).

[22] A. Hasegawa, Plasma Instabilities and Nonlinear Effects, Springer-Verlag, Heidelberg 1975.

[23] L.A. Ostrovskii, Sov. Phys. JETP 24, 797 (1967).

[24] V.I. Bespalov, V.I. Talanov, JETP Lett. 3, 307 (1966); V.I. Karpman, JETP Lett. 6, 277 (1967).

[25] Yu.S. Kivshar, M. Peyrard, Phys. Rev. A 46, 3198 (1992). 
[26] P.D. Miller, O. Bang, Phys. Rev. E 57, 6038 (1998).

[27] M.J. Potasek, Opt. Lett. 12, 921 (1987).

[28] Yu.S. Kivshar, D. Anderson, M. Lisak, Phys. Scr. 48, 679 (1993).

[29] M. Soljacic, M. Segev, T. Coskun, D.N. Christodoulides, A. Vishwanath, Phys. Rev. Lett. 84, 467 (2000).

[30] W. Krolikowski, O. Bang, J.J. Rasmussen, J. Wyller, Phys. Rev. E 64, 016612 (2001)

[31] J.J. Rasmussen, K. Rypdal, Phys, Scr. 33, 481 (1986).

[32] L. Bergé, Phys. Rep. 303, 259 (1998).

[33] Y.S. Kivshar, D.E. Pelinovsky, Phys. Rep. 331, 117 (2000).

[34] A.Y. Wong, P.Y. Cheung, Phys. Rev. Lett. 52, 1222 (1984).

[35] E. Garmire, R.Y. Chiao, C.H. Towns, Phys. Rev. Lett. 16, 347 (1966).

[36] C.A. Sackett, J.M. Gerton, M. Welling, R.G. Hulet, Phys. Rev. Lett. 82, 876 (1999).

[37] P.P. Banerjee, A. Korpel, K.E. Lonngren, Phys. Fluids 26, 2393 (1983).

[38] S.L. Shapiro, S.A. Teukolsky, Black Holes, White Dwarfs, and Neutron Stars The Physics of Compact Objects, Wiley, New York 1983.

[39] M. Houbiers, H.T. Stoof, Phys. Rev. A 54, 5055 (1996).

[40] C. Sulem, P. Sulem, The Nonlinear Schrödinger Equation, Springer-Verlag, Berlin 1999

[41] J.M. Gerton, D. Strekalov, I. Prodan, R.G. Hulet, Nature 408, 692 (2000).

[42] E.A. Donley, N.R. Claussen, S.L. Cornish, J.L. Roberts, E.A. Cornell, C.E. Wieman, Nature 412, 295 (2001).

[43] V.M. Pérez-García, V.V. Konotop, J.J. García-Ripoll, Phys. Rev. E 62, 4300 (2000).

[44] O. Bang, W. Krolikowski, J. Wyller, J.J. Rasmussen, Phys. Rev. E 66, 046619 (2002).

[45] A. Parola, L. Salasnich, L. Reatto, Phys. Rev. A 57, R3180 (1998).

[46] D. Anderson, Phys. Rev. A 27, 3135 (1983).

[47] N.G. Vakhitov, A.A. Kolokolov, Radiophys. Quantum Electron. 16, 783 (1975); A.A. Kolokolov, Radiophys. Quantum Electron. 17, 1016 (1976).

[48] W. Królikowski, O. Bang, Phys. Rev. E 63, 016610 (2001).

[49] I. Bialynicki-Birula, J. Mycielski, Phys. Scr. 20, 539 (1979); S. Cowan, R.H. Enns, S.S. Rangnekar, S.S. Sanghera, Can. J. Phys. 64, 311 (1986); S. Gatz, J. Herrman, Opt. Lett. 17, 484 (1992).

[50] E.W. Ladtke, K.H. Spatschek, L. Stenflo, J. Math. Phys. 24, 2764 (1983).

[51] Y. Kivshar, W. Krolikowski, Opt. Lett. 20, 1527 (1995). 